\title{
Perioperative gait analysis after total hip arthroplasty: Does outpatient surgery compromise patient outcomes?
}

\author{
Bryn O. Zomar, PhD \\ Dianne M. Bryant, PhD \\ Susan W. Hunter, PhD \\ James L. Howard, MD, MSc \\ Brent A. Lanting, MD, MSc
}

Presented at the American Academy of Orthopaedic Surgeons 2018 Annual Meeting, Mar. 6-10, 2018, New Orleans, La.

Accepted Aug. 12, 2020

\section{Correspondence to:}

B. Lanting

Rm B9-003, London Health

Sciences

Centre - University Hospital

339 Windermere Rd E

London ON N6A 5A5

brent.lanting@Ihsc.on.ca

DOI: $10.1503 /$ cjs.008620
Background: There has been a continuing trend toward decreasing the length of hospital stay for patients undergoing total hip arthroplasty (THA). We aimed to investigate the impact of timing of discharge on gait and patient-reported outcomes early after THA.

Methods: In this prospective observational cohort study conducted from May 2014 to November 2015, we measured gait velocity, stride length, single-limb support and single-limb support symmetry in adults aged 18-75 years before direct anterior THA, at discharge from the hospital, and 2, 6 and 12 weeks postoperatively. All procedures were performed by a single surgeon. Patients were discharged on the same day as surgery (outpatient group) or stayed at least 1 night in hospital (inpatient group). Participants also completed the Timed Up and Go test (all postoperative time points) and a series of questionnaires (Western Ontario and McMaster Universities Osteoarthritis Index [6 and 12 wk], 12-Item Short Form Health Survey [2, 6 and 12 wk], Harris Hip Score [12 wk] and a pain visual analogue scale [all postoperative time points]).

Results: Thirty-six participants were enrolled in the study, of whom 16 were outpatients and 20 were inpatients. The mean pain rating at the time of discharge was lower in the outpatient group than in the inpatient group (adjusted mean difference $-1.5,95 \%$ confidence interval -3.0 to 0.0 ). We found no other significant differences between the groups for any gait, patient-reported or surgical outcome.

Conclusion: There were no statistically significant differences in gait or patient-reported outcomes after direct anterior THA between patients who stayed overnight and those who were discharged as outpatients. Patients discharged as outpatients were younger than those who stayed overnight. Our results suggest that discharging patients as an outpatient after direct anterior THA may have a similar impact on patient function and outcomes as a standard overnight stay in hospital.

Contexte : La tendance à réduire la durée de l'hospitalisation des patients soumis à une intervention pour prothèse totale de la hanche (PTH) se maintient. Nous avons voulu évaluer l'impact du moment du congé sur la démarche et sur les paramètres rapportés par les patients peu de temps après la PTH.

Méthodes : Au cours de cette étude de cohorte observationnelle prospective réalisée entre mai 2014 et novembre 2015, nous avons mesuré la vitesse de la déambulation, la longueur de la foulée, l'équilibre sur une jambe et sa symétrie chez des adultes de 18 à 75 ans avant une PTH antérieure directe, au moment du congé hospitalier et 2, 6 et 12 semaines après l'intervention. Toutes les interventions ont été effectuées par 1 seul chirurgien. Les patients recevaient leur congé le jour même de l'opération (groupe de patients ambulatoires) ou séjournaient au moins 1 nuit à l'hôpital (groupe de patients hospitalisés). Les participants effectuaient aussi un test de lever-marcher chronométré (Timed Up and Go test) à toutes les évaluations postopératoires, et on leur administrait une série de questionnaires : score WOMAC (Western Ontario and McMaster Universities Osteoarthritis Index) à 6 et 12 semaines, SF-12 (12-Item Short Form Health Survey) à 2, 6 et 12 semaines, score de hanche de Harris à 12 semaines et échelle visuelle analogique d'intensité de la douleur à toutes les évaluations postopératoires.

Résultats : Trente-six participants ont été inscrits à l'étude, dont 16 dans le groupe ambulatoire et 20 dans le groupe hospitalisé. L'évaluation moyenne de la douleur au moment du congé étaient moindre dans le groupe de patients hospitalisés (différence moyenne ajustée $-1,5$, intervalle de confiance de $95 \%-3,0$ à 0,0$)$. Nous n'avons observé aucune autre différence significative entre les groupes pour la démarche et les paramètres autorapportés ou opératoires.

Conclusion : Nous n'avons observé aucune différence statistiquement significative entre les groupes aux plans de la démarche ou des paramètres autorapportés après la PTH antérieure directe selon que les patients avaient séjourné 1 nuit à l'hôpital ou qu'ils avaient reçu leur congé le jour même. Les patients non hospitalisés étaient plus jeunes que les patients hospitalisés. Selon nos résultats, l'impact sur le fonctionnement et sur les paramètres des patients pourrait être le même, que ces derniers reçoivent leur congé hospitalier le jour même ou le lendemain. 
$\mathrm{T}$ otal hip arthroplasty (THA) is a successful procedure for the treatment of osteoarthritis. Length of stay after this procedure continues to decrease, and some institutions have started to perform THA as an outpatient procedure, discharging patients from hospital on the same day as surgery. This was initially done in the United States but has been implemented in Canada and Europe as well. ${ }^{1}$

Most research thus far has focused almost exclusively on rates of complications and readmissions, with most studies showing similar rates among those discharged as outpatients and those with an overnight stay. ${ }^{2-4}$ Few studies have investigated patient-reported outcomes, and those that have have focused on satisfaction or pain, with reports of high satisfaction and lower or equivalent pain in outpatient cohorts. ${ }^{5-11}$ Hoeffel and colleagues ${ }^{11}$ evaluated patient outcomes using the Oxford Hip Score and Oxford Knee Score and found improvements 12 months postoperatively similar to previously published data on surgical procedures performed in an inpatient hospital setting.

Abnormal gait patterns may persist up to 1 year after THA. ${ }^{12}$ It is therefore important to assess gait in the early postoperative recovery phase to better understand walking patterns and spatiotemporal gait parameters. Comparing gait outcomes between outpatients and inpatients after THA is important to investigate whether increased early functional demands affect gait recovery. In the present study, we aimed to compare early gait outcomes such as gait velocity and symmetry between patients discharged as outpatients after THA and those who stayed at least 1 night in hospital. We also wished to investigate patientreported outcomes including function, quality of life, pain and complications.

\section{Methods}

This was a prospective observational cohort study conducted from May 2014 to November 2015. It was approved by the Western University Health Science Research Ethics Board. We recruited a convenience sample of consecutive patients aged 18-75 years undergoing primary unilateral THA via the direct anterior approach performed by a single surgeon to treat osteoarthritis. Participants were excluded if they had a body mass index greater than 40 , were unable to ambulate at least $10 \mathrm{~m}$ without the use of a gait aid before surgery, had undergone ipsilateral total knee arthroplasty or had other comorbidities of the lower extremities that would affect their gait.

Total hip arthroplasty was performed with the patient in a supine position on a specialized table (Hana, Mizuho OSI). All patients received a periarticular injection (ropivacaine, $300 \mathrm{mg}$, ketorolac tromethamine, $30 \mathrm{mg}$, and morphine, $10 \mathrm{mg}$ ) at the end of the procedure.

The decision as to when to discharge patients to home was made together by the surgeon, nurses and patient based on whether our institution's discharge criteria (including ability to use required gait aids, appropriate pain control, control or absence of nausea and vomiting, alert and oriented, able to use the bathroom, meets the hospital standard targets from physiotherapy, given take-home medications and in the company of a caregiver) had been met.

\section{Outcomes}

Participants completed assessments before surgery, at discharge from the hospital, and at 2, 6 and 12 weeks postoperatively. For the primary outcome of gait function, we used the GAITRite (CIR Systems) to collect velocity, stride length, step length, single-limb support and doublelimb support. ${ }^{13-15}$ The GAITRite is a pressure-sensitive portable walkway consisting of a mat that is $8.3 \mathrm{~m}$ long by $0.9 \mathrm{~m}$ wide. The mat contains embedded sensors that allow for the collection of temporal and spatial data by a computer connected to the mat. Participants were permitted to use whatever gait aid they required to complete the tests.

We used the values for single-limb support and step length to calculate symmetry ratios by dividing the result obtained for the operative limb by that obtained for the nonoperative limb. Perfect symmetry is indicated by a value of 1 . Values less than 1 favour the nonoperative limb for single-limb support and the operative limb for step length, and values greater than 1 favour the operative limb for single-limb support and the nonoperative limb for step length.

We also asked participants to complete the Timed Up and Go test (TUG), Western Ontario and McMaster Universities Osteoarthritis Index (WOMAC), 12-Item Short Form Health Survey (SF-12), Harris Hip Score and visual analogue scale for pain (rated from 0 [no pain] to 10 [worst pain]) to assess function, quality of life and pain. We collected all outcomes before surgery at the preadmission visit. The TUG and pain visual analogue scale were completed at all postoperative time points, the SF-12 was completed at 2, 6 and 12 weeks, the WOMAC was completed at 6 and 12 weeks, and the Harris Hip Score was completed at 12 weeks.

\section{Statistical analysis}

We used descriptive statistics to present the demographic characteristics of the participants using means and standard deviations (SDs) for continuous variables (age, body mass index) and proportions for nominal variables (sex, operative hip, previous hip surgery, dominant side). We presented all continuous data (TUG result, WOMAC score, gait outcomes, visual analogue scale rating, SF-12 score, Harris Hip Score) as mean and standard error (SE), and all comparisons with $95 \%$ confidence intervals (CIs) around the estimate. We analyzed all outcomes using analyses of 
covariance where preoperative assessments were used as a covariate to control for any potential differences between the 2 groups. We performed data analysis using SPSS version 24 software (IBM Corp.).

\section{Results}

Thirty-six patients were enrolled, of whom 16 were discharged on the same day as surgery (outpatients), and 20 stayed at least 1 night in hospital (inpatients). The outpatient cohort was younger $(p=0.01)$ and more likely to have taken prescription pain medication to manage their pain preoperatively $(p=0.04)$ than the inpatient cohort (Table 1).

There were no statistically significant differences between outpatients and inpatients for any of the gait outcomes at any time point (Table 2). The number of participants unable to complete the walk test was not different between the groups at any time point $(p>0.05)$. The outpatient group had less pain at the time of discharge from hospital than the inpatient group $(p=0.04)$ (Table 3$)$. There were no other significant differences in patientreported outcomes between the groups.

There was no statistically significant difference between the groups in gait aid use at any time point. About half of the participants in both groups had stopped using a gait aid by 2 weeks after surgery, and almost all by 6 weeks. Only 1 participant in either group was still using a gait aid at 12 weeks.

There were no statistically significant differences between the groups in any surgical outcome such as duration of surgery, release of the conjoint tendon or time since last pain medication before gait testing. However, there was a greater decrease in hemoglobin concentration in the inpatient group than in the outpatient group $(p=$ 0.001).

There were 6 adverse events in 5 participants in the inpatient group: deep vein thrombosis, diverticulitis, bursitis (2 patients), Clostridium difficile infection and subsidence of the femoral stem at 6 weeks. One patient in the outpatient group fell just before the 12-week appointment; there was no injury to the hip, and no additional treatment was required.

\section{Discussion}

We found no statistically significant differences between the inpatient and outpatients groups in any of the gait parameters that were measured, including velocity, stride length, and single-limb and double-limb support. There were also no statistically significant differences found between the groups for most of the patient-reported outcomes. The outpatient group was younger than the inpatient group and outpatients were more likely than inpatients to be taking pain medications before surgery.
Table 1. Baseline demographic characteristics of patients undergoing total hip arthroplasty through the direct anterior approach discharged as outpatients or inpatients

\begin{tabular}{|lccc} 
& \multicolumn{2}{c}{ No. (\%) of patients* } & \\
\cline { 2 - 3 } & $\begin{array}{c}\text { Inpatients } \\
n=20\end{array}$ & $\begin{array}{c}\text { Outpatients } \\
n=16\end{array}$ & p value \\
\hline Characteristic & $12(60)$ & $9(56)$ & 0.8 \\
\hline Male sex & $64.6 \pm 9.0$ & $56.8 \pm 7.9$ & 0.01 \\
\hline Age, mean \pm SD, yr & $169.2 \pm 10.9$ & $172.0 \pm 9.1$ & 0.4 \\
\hline Height, mean $\pm \mathrm{SD}, \mathrm{cm}$ & $81.1 \pm 13.8$ & $82.6 \pm 15.2$ & 0.8 \\
\hline Weight, mean \pm SD, kg & $28.8 \pm 5.0$ & $27.8 \pm 3.9$ & 0.7 \\
\hline Body mass index, mean \pm SD & $10(50)$ & $8(50)$ & 1.0 \\
\hline Operative hip: left & $2(10)$ & $3(19)$ & 0.4 \\
\hline Symptoms in contralateral hip & $8(40)$ & $12(75)$ & 0.04 \\
\hline Prescription pain medication & $6(30)$ & $6(38)$ & 0.6 \\
\hline Previous hip surgery & $2(10)$ & $1(6)$ & 0.7 \\
\hline Previous spinal surgery & & & \\
\hline $\begin{array}{l}\text { SD }=\text { standard deviation. } \\
\text { *Except where noted otherwise. }\end{array}$ & & & \\
\hline
\end{tabular}

Outpatients also reported less pain on the day of discharge; however, this difference was small and likely not clinically important, as the upper bound of the CI included 0 . We also found a higher complication rate in the inpatient group than in the outpatient group.

A 2016 systematic review by Pollock and colleagues ${ }^{4}$ showed no difference in complication rates between outpatient and inpatient THA groups, but costs were markedly lower for outpatients. However, those authors stated that the overall quality of the studies included in their analysis was weak and that higher-quality, randomized studies are needed to adequately assess the safety and feasibility of outpatient pathways.

Goyal and colleagues ${ }^{7}$ conducted a multicentre randomized study in the US and found results contrary to ours, with outpatients reporting greater pain early after surgery and no differences later. They also found that patients allocated to the inpatient group who elected to leave early as outpatients were younger than those who stayed overnight. Major weaknesses of this study were a lack of blinding and of enforcement of group allocation, with the result that only about $75 \%$ of participants were discharged as randomly allocated. ${ }^{7}$

Dorr and colleagues ${ }^{8}$ had patients keep a diary of functional milestones reached in the first 3 weeks postoperatively. They reported mean pain scores at 2 weeks that were similar to those reported by our outpatient group (2.5 [SD 1.7] v. 2.6 [SE 0.5]); however, they reported a lower proportion of patients walking independently without use of a gait aid at the same time point ( $41 \%$ v. $50 \%)$.

The younger age of the outpatients in the present study is not surprising, as most prospective studies on outpatient THA usually include upper age limits in their eligibility criteria. ${ }^{16}$ Although a range of age limits has been reported in the literature, many investigators cite increased risks of complications and readmission in patients older than 
Table 2. Temporal and spatial gait characteristics for the 2 groups

\begin{tabular}{|c|c|c|c|c|}
\hline \multirow[b]{2}{*}{ Characteristic } & \multirow[b]{2}{*}{ Time } & \multicolumn{2}{|c|}{ Mean \pm SE } & \multirow{2}{*}{$\begin{array}{l}\text { Adjusted mean difference } \\
\qquad(95 \% \mathrm{Cl})\end{array}$} \\
\hline & & Inpatients & Outpatients & \\
\hline \multirow[t]{5}{*}{ Velocity, cm/s } & Preoperative & $98.8 \pm 6.2$ & $99.9 \pm 5.2$ & $1.1(-15.8$ to 18.0$)$ \\
\hline & Discharge & $32.0 \pm 4.8$ & $41.7 \pm 5.1$ & $9.7(-4.5$ to 23.8$)$ \\
\hline & $2 w k$ & $83.3 \pm 5.9$ & $86.4 \pm 6.7$ & $3.1(-15.0$ to 21.3$)$ \\
\hline & $6 w k$ & $109.2 \pm 3.9$ & $109.8 \pm 4.4$ & $0.6(-11.5$ to 12.6$)$ \\
\hline & $12 w k$ & $114.9 \pm 3.4$ & $116.8 \pm 3.8$ & $1.9(-8.5$ to 12.4$)$ \\
\hline \multirow[t]{5}{*}{ Stride length, cm } & Preoperative & $114.2 \pm 5.7$ & $118.6 \pm 4.7$ & $4.5(-11.1$ to 20.0$)$ \\
\hline & Discharge & $66.0 \pm 4.7$ & $76.7 \pm 5.0$ & $10.7(-3.2$ to 24.6$)$ \\
\hline & $2 w k$ & $106.3 \pm 3.9$ & $107.5 \pm 4.4$ & $1.1(-10.9$ to 13.1$)$ \\
\hline & $6 w k$ & $124.0 \pm 3.4$ & $124.8 \pm 3.8$ & $0.8(-9.5$ to 11.2$)$ \\
\hline & 12 wk & $129.3 \pm 2.6$ & $130.5 \pm 2.9$ & $1.2(-6.9$ to 9.2$)$ \\
\hline \multirow{5}{*}{$\begin{array}{l}\text { Double-limb support, \% of gait } \\
\text { cycle }\end{array}$} & Preoperative & $30.7 \pm 1.1$ & $30.3 \pm 1.1$ & $-0.4(-3.5$ to 2.7$)$ \\
\hline & Discharge & $58.5 \pm 3.2$ & $51.4 \pm 3.4$ & $-7.1(-16.8$ to 2.5$)$ \\
\hline & $2 w k$ & $34.8 \pm 1.8$ & $34.9 \pm 2.0$ & $0.1(-5.3$ to 5.5$)$ \\
\hline & $6 w k$ & $29.7 \pm 0.8$ & $29.6 \pm 0.9$ & $-0.1(-2.4$ to 2.3$)$ \\
\hline & $12 w k$ & $28.8 \pm 0.7$ & $27.6 \pm 0.7$ & $-1.2(-3.2$ to 0.8$)$ \\
\hline \multicolumn{5}{|l|}{$\begin{array}{l}\text { Single-limb support, \% of gait } \\
\text { cycle }\end{array}$} \\
\hline \multirow[t]{5}{*}{ Operative limb } & Preoperative & $33.2 \pm 0.7$ & $34.1 \pm 0.7$ & $0.9(-1.3$ to 3.1$)$ \\
\hline & Discharge & $20.3 \pm 1.6$ & $22.2 \pm 1.7$ & $2.0(-3.0$ to 6.9$)$ \\
\hline & $2 w k$ & $31.3 \pm 1.2$ & $30.9 \pm 1.4$ & $-0.4(-4.1$ to 3.3$)$ \\
\hline & $6 w k$ & $35.0 \pm 0.6$ & $35.0 \pm 0.7$ & $0.0(-1.8$ to 1.9$)$ \\
\hline & $12 w k$ & $35.5 \pm 0.5$ & $36.6 \pm 0.5$ & $1.1(-0.3$ to 2.5$)$ \\
\hline \multirow[t]{5}{*}{ Nonoperative limb } & Preoperative & $36.0 \pm 0.5$ & $35.7 \pm 0.5$ & $-0.3(-1.6$ to 1.1$)$ \\
\hline & Discharge & $23.7 \pm 1.4$ & $26.9 \pm 1.5$ & $3.3(-0.9$ to 7.4$)$ \\
\hline & $2 w k$ & $33.8 \pm 0.7$ & $34.2 \pm 0.8$ & $0.3(-1.8$ to 2.4$)$ \\
\hline & $6 w k$ & $35.6 \pm 0.3$ & $35.6 \pm 0.4$ & $-0.1(-1.1$ to 1.0$)$ \\
\hline & 12 wk & $36.1 \pm 0.4$ & $36.0 \pm 0.4$ & $0.0(-1.2$ to 1.1$)$ \\
\hline \multicolumn{5}{|l|}{ Step length, cm } \\
\hline \multirow[t]{5}{*}{ Operative limb } & Preoperative & $58.2 \pm 3.0$ & $60.3 \pm 2.5$ & $2.1(-6.2$ to 10.3$)$ \\
\hline & Discharge & $43.1 \pm 2.4$ & $45.8 \pm 2.5$ & $2.7(-4.4$ to 9.9$)$ \\
\hline & $2 w k$ & $56.4 \pm 1.6$ & $59.1 \pm 1.8$ & $2.7(-2.2$ to 7.6$)$ \\
\hline & $6 \mathrm{wk}$ & $63.0 \pm 1.5$ & $63.2 \pm 1.7$ & $0.2(-4.4$ to 4.8$)$ \\
\hline & $12 \mathrm{wk}$ & $65.3 \pm 1.2$ & $66.7 \pm 1.3$ & $1.4(-2.2$ to 5.1$)$ \\
\hline \multirow[t]{5}{*}{ Nonoperative limb } & Preoperative & $55.8 \pm 2.7$ & $58.0 \pm 2.2$ & $2.1(-5.3$ to 9.6$)$ \\
\hline & Discharge & $22.8 \pm 3.9$ & $30.9 \pm 4.2$ & $8.2(-3.5$ to 19.8$)$ \\
\hline & $2 w k$ & $49.7 \pm 2.9$ & $48.6 \pm 3.2$ & $-1.1(-9.9$ to 7.7$)$ \\
\hline & $6 w k$ & $60.7 \pm 2.0$ & $61.5 \pm 2.2$ & $0.8(-5.2$ to 6.8$)$ \\
\hline & $12 w k$ & $63.6 \pm 1.5$ & $63.5 \pm 1.6$ & $-0.1(-4.6$ to 4.4$)$ \\
\hline \multirow[t]{5}{*}{ Step length, symmetry ratio* } & Preoperative & $1.04 \pm 0.02$ & $1.04 \pm 0.02$ & $0.00(0.03$ to -0.06$)$ \\
\hline & Discharge & $6.33 \pm 1.61$ & $2.96 \pm 1.70$ & $-3.36(-8.14$ to 1.41$)$ \\
\hline & $2 w k$ & $1.19 \pm 0.14$ & $1.40 \pm 0.16$ & $0.21(-0.21$ to 0.63$)$ \\
\hline & 6 wk & $1.05 \pm 0.02$ & $1.04 \pm 0.02$ & $-0.02(-0.07$ to 0.04$)$ \\
\hline & $12 \mathrm{wk}$ & $1.04 \pm 0.01$ & $1.05 \pm 0.01$ & $0.01(-0.02$ to 0.05$)$ \\
\hline \multirow{5}{*}{$\begin{array}{l}\text { Single-limb support, symmetry } \\
\text { ratio* }\end{array}$} & Preoperative & $0.92 \pm 0.02$ & $0.95 \pm 0.02$ & $0.03(-0.02$ to 0.08$)$ \\
\hline & Discharge & $0.83 \pm 0.04$ & $0.83 \pm 0.04$ & $0.00(-0.11$ to 0.11$)$ \\
\hline & $2 w k$ & $0.92 \pm 0.03$ & $0.91 \pm 0.03$ & $-0.01(-0.09$ to 0.08$)$ \\
\hline & $6 w k$ & $0.98 \pm 0.02$ & $0.99 \pm 0.02$ & $0.01(-0.04$ to 0.06$)$ \\
\hline & $12 \mathrm{wk}$ & $0.98 \pm 0.01$ & $1.02 \pm 0.02$ & 0.04 (0.00 to 0.08$)$ \\
\hline \multirow[t]{5}{*}{ Timed Up and Go test, s } & Preoperative & $11.00 \pm 0.86$ & $10.77 \pm 1.07$ & $-0.23(-2.99$ to 2.52$)$ \\
\hline & Discharge & $48.43 \pm 3.20$ & $39.25 \pm 3.40$ & $-9.18(-18.71$ to 0.35$)$ \\
\hline & $2 w k$ & $14.99 \pm 2.01$ & $15.02 \pm 2.24$ & $0.03(-6.10$ to 6.16$)$ \\
\hline & $6 w k$ & $9.65 \pm 0.50$ & $9.44 \pm 0.56$ & $-0.21(-1.73$ to 1.32$)$ \\
\hline & $12 w k$ & $9.35 \pm 0.42$ & $8.54 \pm 0.47$ & $-0.81(-2.09$ to 0.48$)$ \\
\hline
\end{tabular}




\begin{tabular}{|c|c|c|c|c|}
\hline \multirow[b]{2}{*}{ Assessment } & \multirow[b]{2}{*}{ Time } & \multicolumn{2}{|c|}{ Mean score \pm SE } & \multirow{2}{*}{$\begin{array}{c}\text { Adjusted mean difference } \\
\qquad(95 \% \mathrm{Cl})\end{array}$} \\
\hline & & Inpatients & Outpatients & \\
\hline \multicolumn{5}{|l|}{ WOMAC } \\
\hline \multirow[t]{3}{*}{ Pain } & Preoperative & $50.8 \pm 4.0$ & $46.6 \pm 3.3$ & $-4.2(-15.1$ to 6.7$)$ \\
\hline & $6 w k$ & $73.9 \pm 3.6$ & $74.0 \pm 4.0$ & $0.1(-10.9$ to 11.1$)$ \\
\hline & $12 w k$ & $83.1 \pm 3.7$ & $84.0 \pm 4.2$ & $0.9(-10.5$ to 12.4$)$ \\
\hline \multirow[t]{3}{*}{ Stiffness } & Preoperative & $46.9 \pm 4.5$ & $39.1 \pm 5.2$ & $-7.8(-21.8$ to 6.2$)$ \\
\hline & $6 \mathrm{wk}$ & $64.7 \pm 4.3$ & $63.8 \pm 4.8$ & $-1.0(-14.2$ to 12.3$)$ \\
\hline & $12 w k$ & $74.7 \pm 4.2$ & $73.8 \pm 4.8$ & $-0.9(-13.9$ to 12.2$)$ \\
\hline \multirow[t]{3}{*}{ Function } & Preoperative & $47.9 \pm 4.2$ & $46.0 \pm 3.5$ & $-1.9(-13.3$ to 9.5$)$ \\
\hline & $6 w k$ & $74.0 \pm 3.4$ & $74.2 \pm 3.8$ & $0.1(-10.2$ to 10.5$)$ \\
\hline & $12 w k$ & $82.0 \pm 3.6$ & $83.2 \pm 4.0$ & $1.3(-9.7$ to 12.2$)$ \\
\hline \multirow[t]{3}{*}{ Total } & Preoperative & $48.9 \pm 4.0$ & $44.8 \pm 3.6$ & $-4.1(-15.3$ to 7.1$)$ \\
\hline & $6 w k$ & $72.0 \pm 3.3$ & $71.9 \pm 3.7$ & $-0.2(-10.3$ to 10.0$)$ \\
\hline & $12 w k$ & $80.8 \pm 3.4$ & $81.6 \pm 3.9$ & 0.8 (-9.8 to 11.4$)$ \\
\hline \multicolumn{5}{|l|}{ SF-12 } \\
\hline \multirow[t]{4}{*}{ Physical component } & Preoperative & $33.7 \pm 2.3$ & $33.0 \pm 2.2$ & $-0.7(-7.4$ to 5.9$)$ \\
\hline & $2 w k$ & $31.1 \pm 1.8$ & $31.5 \pm 2.0$ & $0.4(-5.0$ to 5.9$)$ \\
\hline & $6 w k$ & $42.3 \pm 2.4$ & $40.1 \pm 2.7$ & $-2.2(-9.4$ to 5.1$)$ \\
\hline & $12 w k$ & $45.3 \pm 2.3$ & $45.6 \pm 2.5$ & 0.26 (-6.7 to 7.2$)$ \\
\hline \multirow[t]{4}{*}{ Mental component } & Preoperative & $55.7 \pm 2.7$ & $54.6 \pm 3.0$ & $-1.1(-9.3$ to 7.0$)$ \\
\hline & $2 w k$ & $50.9 \pm 2.3$ & $56.8 \pm 2.6$ & $5.9(-1.2$ to 13.0$)$ \\
\hline & $6 w k$ & $54.1 \pm 2.0$ & $54.5 \pm 2.2$ & $0.3(-5.7$ to 6.4$)$ \\
\hline & $12 w k$ & $55.9 \pm 1.6$ & $56.9 \pm 1.8$ & $1.1(-4.0$ to 6.1$)$ \\
\hline \multirow[t]{2}{*}{ Harris Hip Score } & Preoperative & $64.1 \pm 2.0$ & $62.8 \pm 2.1$ & $-1.3(-7.2$ to 4.7$)$ \\
\hline & $12 \mathrm{wk}$ & $95.8 \pm 1.2$ & $96.3 \pm 1.3$ & 0.6 (-3.1 to 4.2$)$ \\
\hline \multirow[t]{5}{*}{ Pain visual analogue scale* } & Preoperative & $5.0 \pm 0.5$ & $5.8 \pm 0.6$ & 0.8 (-0.8 to 2.4$)$ \\
\hline & Discharge & $4.6 \pm 0.5$ & $3.1 \pm 0.5$ & $-1.5(-3.0$ to 0.0$)$ \\
\hline & $2 w k$ & $2.5 \pm 0.5$ & $2.6 \pm 0.5$ & 0.0 (-1.4 to 1.5$)$ \\
\hline & $6 w k$ & $1.2 \pm 0.5$ & $1.6 \pm 0.6$ & $0.4(-1.2$ to 2.0$)$ \\
\hline & $12 w k$ & $0.6 \pm 0.4$ & $1.0 \pm 0.4$ & $0.3(-0.9$ to 1.5$)$ \\
\hline
\end{tabular}

80 years of age. ${ }^{16}$ Gromov and colleagues ${ }^{17}$ investigated the feasibility of discharging an unselected group of patients on the same day as surgery and found that, compared to younger patients, those aged older than 75 had 2.6 times greater odds of being unfit for discharge.

Although we found a statistically significant difference in pain ratings between groups on the day of discharge, with outpatients reporting less pain than inpatients, it is possible that this difference was due to the timing of data collection. Patients discharged as outpatients were asked to assess their pain only hours after surgery, when the periarticular injection administered at the end of the procedure was likely in full effect, whereas inpatients were asked on postoperative day 1 or 2, after the effects of the injection would have worn off. The study was also underpowered to detect such a difference, and therefore it is possible that the difference we found is a type I error. The CI around the estimate of pain on the day of discharge is quite large, with the lower limit including a possibly clinically important between-group difference (reported as about $20 \%$ of the within-group minimally clinically important difference, ${ }^{18}$ suggested to be $\left.1.10-1.37\right) .{ }^{19}$ However, the upper limit of our CI includes 0 , which suggests that there may have been no difference in pain between the groups. A larger sample would help to determine whether there is a difference in pain on the day of discharge between patients discharged as outpatients and those who stay at least 1 night in hospital.

Our inpatient group experienced a greater postoperative decrease in hemoglobin concentration than the outpatient group. We suspect that this difference may be attributable to the timing of measurement. The postoperative blood draw occurred at different times for the 2 groups: all outpatients had their postoperative blood draw before leaving the hospital, on the same day as their procedure, whereas all inpatients had their blood drawn on postoperative day 1 . It is possible that the outpatients experienced a similar change in hemoglobin concentration as the inpatients but that this was not observed because their blood draw occurred before the change. 
At discharge, the outpatient group seemed to perform better than the inpatient group for the gait parameters measured; however, the differences did not reach statistical significance. There may have been significant or clinically important differences between the groups, as both the upper and lower limits of the CIs included potentially clinically important differences in favour of either group. For example, for gait velocity, it has been reported that the within-group minimally clinically important difference is between 0.08 and $0.26 \mathrm{~m} / \mathrm{s}^{20}$ With a between-group difference about $20 \%$ of the within-group difference, ${ }^{19}$ differences as small as $0.016 \mathrm{~m} / \mathrm{s}-0.052 \mathrm{~m} / \mathrm{s}$ between groups may be clinically important. Our CIs at all time points included differences larger than this, which suggests that there may have been an important difference between the groups that our study was unable to detect. It is also possible that the larger differences observed between the groups at discharge than at other time points may have been due to the timing of testing, as all outpatients performed the gait tests while still feeling the effects of the periarticular injection.

\section{Limitations}

Limitations of our study include the small sample and lack of randomization. However, the consistency of our findings across the various function and patient-reported outcomes strengthens our conclusions. Confidence intervals around the mean differences were quite wide, which reflects our imprecision. Increasing our sample size would help to improve the precision in our estimates and provide more confidence in our conclusions.

Lack of randomization contributed to differences in baseline characteristics such as age between our groups. In future studies, it would be important to include randomized group assignment to ensure that groups are comparable so that any differences observed can be confidently attributed to the timing of discharge and not underlying group characteristics.

A strength of our study is the assessment of gait function at multiple early time points, including on the day of discharge from the hospital.

\section{Conclusion}

We found no statistically significant differences in gait or patient-reported outcomes between patients discharged from hospital as outpatients versus inpatients after direct anterior THA. It is possible that, owing to our small sample, significant differences between the groups may have been missed. Patients in the outpatient group were younger than those who stayed at least 1 night in hospital. Our results suggest that discharging patients as an outpatient after direct anterior THA may have a similar impact on patient function and outcomes as a standard overnight stay in hospital.
Affiliations: From the Faculty of Health Sciences, Western University, London, Ont. (Zomar, Bryant, Hunter); the Bone and Joint Institute, Western University, London, Ont. (Zomar, Bryant); the London Health Sciences Centre, London, Ont. (Howard, Lanting); and the Schulich School of Medicine and Dentistry, Western University, London, Ont. (Howard, Lanting).

Competing interests: James Howard reports grants from Stryker and DePuy Synthes, personal fees from Stryker, DePuy Synthes, Smith \& Nephew and Intellijoint Surgical, and institutional research support from Stryker, DePuy Synthes, Smith \& Nephew, Zimmer Biomet and MicroPort, outside the submitted work. He holds stock in PersaFix Technologies. Brent Lanting reports grants and personal fees from DePuy Synthes, Stryker and Smith \& Nephew, and institutional support from DePuy Synthes, Stryker, Smith \& Nephew and Zimmer Biomet, outside the submitted work. No other competing interests were declared.

Contributors: B. Zomar, D. Bryant, J. Howard and B. Lanting designed the study. B. Zomar acquired the data, which B. Zomar, D. Bryant, S. Hunter and B. Lanting analyzed. B. Zomar wrote the article, which D. Bryant, S. Hunter, J. Howard and B. Lanting critically reviewed. All authors approved the article for publication.

Content licence: This is an Open Access article distributed in accordance with the terms of the Creative Commons Attribution (CC BYNC-ND 4.0) licence, which permits use, distribution and reproduction in any medium, provided that the original publication is properly cited, the use is noncommercial (i.e., research or educational use), and no modifications or adaptations are made. See: https://creativecommons. org/licenses/by-nc-nd/4.0/.

\section{References}

1. Outpatient (same-day) total hip replacement. CADTH Issues in Emerging Health Technologies 2017;152 March.

2. Lovett-Carter D, Sayeed Z, Abaab L, et al. Impact of outpatient total joint replacement on postoperative outcomes. Orthop Clin North Am 2018;49:35-44.

3. Hoffmann JD, Kusnezov NA, Dunn JC, et al. The shift to same-day outpatient joint arthroplasty: a systematic review. 7 Artbroplasty 2018; 33:1265-74.

4. Pollock M, Somerville L, Firth A, et al. Outpatient total hip arthroplasty, total knee arthroplasty, and unicompartmental knee arthroplasty: a systematic review of the literature. $7 B 7 S$ Rev 2016;4: 01874474-201612000-00004.

5. Larsen JR, Skovgaard B, Prynø T, et al. Feasibility of day-case total hip arthroplasty: a single-centre observational study. Hip Int 2017;27: 60-5.

6. Kelly MP, Calkins TE, Culvern C, et al. Inpatient versus outpatient hip and knee arthroplasty: Which has higher patient satisfaction? $\mathcal{f}$ Arthroplasty 2018;33:3402-6.

7. Goyal N, Chen AF, Padgett SE, et al. Otto Aufranc Award: a multicenter, randomized study of outpatient versus inpatient total hip arthroplasty. Clin Orthop Relat Res 2017;475:364-72.

8. Dorr LD, Thomas DJ, Zhu J, et al. Outpatient total hip arthroplasty. 7 Arthroplasty 2010;25:501-6.

9. Berend ME, Lackey WG, Carter JL. Outpatient-focused joint arthroplasty is the future: the Midwest Center for Joint Replacement experience. 7 Arthroplasty 2018;33:1647-8.

10. Paredes O, Núñez R, Klaber I. Successful initial experience with a novel outpatient total hip arthroplasty program in a public health system in Chile. Int Orthop 2018;42:1783-7.

11. Hoeffel DP, Daly PJ, Kelly BJ, et al. Outcomes of the first 1,000 total hip and total knee arthroplasties at a same-day surgery center using a rapid-recovery protocol. 7 Am Acad Orthop Surg Glob Res Rev 2019;3:e022. 
12. Ewen AM, Stewart S, St Clair Gibson A, et al. Post-operative gait analysis in total hip replacement patients - a review of current literature and meta-analysis. Gait Posture 2012;36:1-6.

13. Bilney B, Morris M, Webster K. Concurrent related validity of the GAITRite ${ }^{\circledR}$ walkway system for quantification of the spatial and temporal parameters of gait. Gait Posture 2003;17: 68-74.

14. Menz HB, Latt MD, Tiedemann A, et al. Reliability of the GAITRite ${ }^{\circledR}$ walkway system for the quantification of temporospatial parameters of gait in young and older people. Gait Posture 2004;20:20-5.

15. McDonough AL, Batavia M, Chen FC, et al. The validity and reliability of the GAITRite system's measurements: a preliminary evaluation. Arch Phys Med Rebabil 2001;82:419-25.

16. Kort NP, Bemelmans YFL, van der Kuy PHM, et al. Patient selection criteria for outpatient joint arthroplasty. Knee Surg Sports Traumatol Artbrosc 2017;25:2668-75.
17. Gromov K, Kjaersgaard-Andersen P, Revald P, et al. Feasibility of outpatient total hip and knee arthroplasty in unselected patients. Acta Orthop 2017;88:516-21.

18. Goldsmith $\mathrm{CH}$, Boers $\mathrm{M}$, Bombardier $\mathrm{C}$, et al. Criteria for clinically important changes in outcomes: development, scoring and evaluation of rheumatoid arthritis patient and trial profiles. F Rheumatol 1993; 20:561-5.

19. Hawker GA, Mian S, Kendzerska T, et al. Measures of adult pain: Visual Analog Scale for Pain (VAS Pain), Numeric Rating Scale for Pain (NRS Pain), McGill Pain Questionnaire (MPQ), Short-Form McGill Pain Questionnaire (SF-MPQ), Chronic Pain Grade Scale (CPGS), Short Form-36 Bodily Pain Scale (SF-36 BPS), and Measure of Intermittent and Constant Osteoarthritis Pain (ICOAP). Arthritis Care Res (Hoboken) 2011;63:S240-52.

20. Bohannon RW, Glenney SS. Minimal clinically important difference for change in comfortable gait speed of adults with pathology: a systematic review. 7 Eval Clin Pract 2014;20:295-300. 